\title{
Physiologic modulation of natural killer cell activity as an index of Alzheimer's disease progression
}

\author{
Paolo Prolo $^{1^{*}}$, Francesco Chiappelli ${ }^{1}$, Alberto Angeli ${ }^{2}$, Andrea Dovio ${ }^{2}$, Paola Perotti ${ }^{2}$, Marisa Pautasso ${ }^{3}$, Maria Luisa Sartori ${ }^{2}$, Laura \\ Saba $^{2}$, Stefano Mussino ${ }^{2}$, Thomas Fraccalini ${ }^{4}$, Fausto Fantó ${ }^{4}$, Cristina Mocellini ${ }^{5}$, Maria Gabriella Rosso ${ }^{5}$ and Enzo Grasso $^{5}$ \\ ${ }^{1}$ Division of Oral Biology \& Medicine, UCLA School of Dentistry, Los Angeles, CA 90095-1668, West-Los Angeles, Veterans \\ Administration Medical Center, Los Angeles, CA 90025; ${ }^{2}$ Internal Medicine, University of Turin, A.S.O. S. Luigi Gonzaga, Orbassano, \\ Italy; ${ }^{3}$ Flow Cytometry Lab, University of Turin, A.S.O. S. Luigi Gonzaga, Orbassano, Italy; ${ }^{4} \mathrm{Geriatrics}$, University of Turin, A.S.O. S. \\ Luigi Gonzaga, Orbassano, Italy; ${ }^{5}$ Neurology, A.S.O. S. Croce e Carle, Cuneo, Italy; Paolo Prolo*- Email: pprolo@dent.ucla.edu; \\ * Corresponding Author \\ received March 14, 2007; accepted March 20, 2006; published online March 21, 2007
}

\begin{abstract}
:
Patients with Alzheimer's disease (AD) are characterized by an altered sensitivity to cortisol-mediated modulation of circulating lymphocytes. Longitudinal studies are needed to address the clinical applicability of these abnormalities as prognostic factors. Therefore, we designed a longitudinal study to address the clinical applicability of physiologic modulation of Natural Killer (NK) cell activity as a prognostic factor in $\mathrm{AD}$. NK activity was assessed as baseline measurement and in response to modulation by cortisol at $10^{-6} \mathrm{M}$. To verify the immunophysiological integrity of the NK cell population, we tested augmentation of NK cytotoxicity by human recombinant interleukin (IL)-2 (100 IU/ml) as control. The response to modulation by cortisol or by IL-2 was significantly greater in patients with AD. Based on change in the Mini-Mental State score at entry and at 18 months, patients with AD could be assigned to a "fast progression" ( $\Delta>2$ points) or to a "slow progression" group $(\Delta \leq 2$ points). The change in the response of NK cytotoxic activity to cortisol, and the strength of the association of this parameter with circulating activated T cells in time was greater in patients with Fast Progression vs. Slow Progression AD. These results suggest that changes in the response of NK cells to negative (e.g., cortisol) or positive modifiers (e.g., IL-2) follow progression of $\mathrm{AD}$.
\end{abstract}

Key Words: Alzheimer's disease (AD); natural killer (NK) cells; NK cytotoxic activity; cortisol; interleukin (IL)-2; psychoimmunology

\section{Background:}

Alzheimer's disease (AD) is a progressive disease of the brain caused by the deposit of $\beta$ Amyloid protein. It is the most common type of dementia in the elderly. By 2010, over five million people will be diagnosed with $\mathrm{AD}$ in the US alone. The social and the medical costs of $\mathrm{AD}$ are mounting rapidly. Current interventions for AD include acetylcholinesterase inhibitors (e.g., Donezepil, [Aricept $\left.{ }^{\circledR}\right]$ ) that are indicated for patients with early symptoms of disease. $[1,2,3]$ Recent data indicate a significant involvement of the T cell-mediated $[4,5]$, as well as the inflammatory arm $[5,6,7]$ of the immune system, which appears to be correlated to the severity of $\mathrm{AD}$. [5, 8] Both in vivo and in vitro studies have demonstrated individual differences, which can be attributed to variables specific to each individual patient.

NK cells, defined immunophenotypically as devoid of the CD3/T cell receptor complex, but expressing the Fc receptor site, CD16, as well as the NK receptor CD56 (CD3-CD16+CD56+), are cytotoxic immune cells that do not require major histocompatibility complex (MHC) recognition. Dysregulations in NK responses are modulated by adaptive physiological processes, i.e., allostasis [5] that may be intrinsic to immune regulation (e.g., IL-2), or that may involve nonimmune factors, such as products of the neuroendocrine system. [9]

Patients with AD exhibit baseline NK activity similar to age- and sex-matched control subjects. However, the altered responsiveness to cortisol modulation of NK activity suggests that patients with $\mathrm{AD}$ may be characterized by a greater sensitivity to cortisol-mediated modulation of circulating lymphocytes. Hence, we propose the

ISSN 0973-2063

Bioinformation 1(9): 363-366 (2007) hypothesis that lack of sensitivity to cortisol modulation of NK activity could have clinically relevant applicability as a prognostic factor of AD. [8]

\section{Description:}

A longitudinal prospective cohort study was designed to address the clinical applicability of NK cell activity as prognostic factors in AD.

NK cell activity of peripheral blood mononuclear (PBM) cells was measured in 18 patients (age range; 61-84 years old) with mild senile dementia of Alzheimer's type (AD), and 10 normal healthy control subjects (four men, six women). Patients were selected on the basis of short history of disease and no medication at baseline. Following diagnosis, all patients were treated with the acetylcholinesterase inhibitor Donezepil $(10 \mathrm{mg} /$ daily $)$. Patients and subjects signed Informed Consent approved by the Institutional Review Board at our institution.

All subjects were monitored at entry $(\mathrm{T}=0)$ and at six-month intervals. At each visit, patients underwent a battery of psychometric tests [Mini-Mental State Examination (MMSE); Mini-Mental State Examination-age and education corrected (MMSE-C); Functional Assessment Staging (FAST); index of Independence in Activities of Daily Living (ADL); Center for Epidemiology Studies-Depression (CES-D); Profile of Moods States (POMS)] to evaluate their cognitive performance and their psychological status. Based on change in the MMSE-C score at 
entry and at 18 months follow-up, patients with AD were assigned to a "fast progression" ( $\Delta>2$ points; $n=8$, two men, six women) or to a "slow progression" group ( $\Delta \leq 2$ points; ( $n=10$; two men, eight women).

NK activity of PBMC incubated with or without modifiers was expressed as Lytic Units (LU) $/ 10^{7}$ cells. [8-11] The summary data were expressed as means \pm standard deviation (SD), or standard error of the mean (SEM), as indicated in the text. Parametric tests were used when the data verified the three assumptions of normal distribution, independence of measurements and homogeneity of variance. When these assumptions were violated, non-parametric tests were used. Statistical significance of differences in the values of cytotoxicity recorded under different conditions by the same PBM cells was determined by paired Student's $t$-test (or Wilcoxon's rank sign test). Differences between levels of cytotoxicity recorded under the same experimental condition in subjects and controls were statistically validated by unpaired two sample $t$-test (or rank sum test). Repeated measure ANOVA (or Friedmann) was used to analyze the longitudinal data. Statistical analysis was performed at a level of significance, $\alpha$, of 0.10 due to the variability in NK activity customarily accounted for by individual differences, using Statistix (NH Analytical Software, USA) softwares and Analyze-It, version 1.72 (Analyze-It Software, Ltd.).

Patients with $\mathrm{AD}$ were distinguished based on the pattern of progression of AD symptoms. Patients with Fast Progression AD exhibit a significant drop in score of MMSE-C (Figure 1a). Figure $1 \mathrm{~b}$ confirms these patterns with the ADL.

The data in Table 1 show comparisons of the cortisol effect on modulating NK activity in the group of normal subjects, and in patients with $\mathrm{AD}$ assigned to a group of fast or slow progressing symptoms. The data in the table indicate that as early as entry, a distinction can be made with respect to what group of patient is likely to progress faster in $\mathrm{AD}$ symptomatology, based on NK activity and on the response of NK cytotoxic activity to cortisol inhibitory and to IL-2 enhancing modulation.

\begin{tabular}{|c|c|c|c|c|c|c|}
\hline & \multicolumn{2}{|c|}{ Baseline } & \multicolumn{2}{|c|}{ Cortisol $\left(10^{-6} \mathrm{M}\right)$} & \multicolumn{2}{|c|}{ HrIL-2 (650 IU/ml) } \\
\hline & $\begin{array}{c}\text { Mean } \\
\text { (lytic units) }\end{array}$ & \pm SEM & $\begin{array}{c}\text { Mean } \\
\text { (lytic units) }\end{array}$ & \pm SEM & $\begin{array}{c}\text { Mean } \\
\text { (lytic units) }\end{array}$ & \pm SEM \\
\hline Control & 32.51 & 6.5 & 23.92 & 3.83 & 75.83 & 19.41 \\
\hline Slow Progressing $\mathrm{AD}^{1}$ & 23.26 & 3.31 & 15.89 & 2.38 & 79.39 & 28.50 \\
\hline$p$ vs. control & 0.24 & & 0.10 & & 0.92 & \\
\hline Fast Progressing $\mathrm{AD}^{1}$ & 21.13 & 3.40 & 15.33 & 2.60 & 38.46 & 6.82 \\
\hline$p$ vs. control & 0.14 & & 0.08 & & 0.09 & \\
\hline
\end{tabular}

Table 1: Modulation of NK Activity (at entry) (1: as determined by drop in MMSE score at 18 months)

The pattern of change of the modulatory response of NK activity in time is shown in Figure 1c and 1d as the median group percent change from baseline untreated cells to cells treated with the physiological modulator. Fig. 1c gives the change in NK modulation to cortisol among normal controls and patients with Slow Progression and Fast Progression AD at six-month intervals between entry and 18 month. The trend of change for the control group can be expressed by the linear equation: $y=-1.3971 x-22.64$, $\mathrm{R}^{2}=0.2223$. The trend of change for the Slow Progression AD patient group can be given by the linear equation: $y=-0.9193 x-$ $34.029, \mathrm{R}^{2}=0.2296$. The trend of change for the Fast Progression $\mathrm{AD}$ patient group is represented by the distinctly different linear equation: $\mathrm{y}=0.302 \mathrm{x}-31.41, \mathrm{R}^{2}=0.2664$.

Fig. 1d shows the pattern of change in NK modulation by IL-2 among normal controls and patients with Slow Progression and Fast Progression $\mathrm{AD}$ at six-month intervals between entry and 18 month. The trend of change for the control group can be expressed by the logarithmic equation: $y=24.777 \operatorname{Ln}(x)+147.25, \mathrm{R}^{2}=0.3606$. The trend of change for the Slow Progression AD patient group is represented by the logarithmic equation: $\mathrm{y}=-69.487 \operatorname{Ln}(\mathrm{x})+$ 229.02, $\mathrm{R}^{2}=0.7786$. The trend of change for the Fast Progression $\mathrm{AD}$ patient group is given by the essentially similar logarithmic equation: $\mathrm{y}=81.205 \operatorname{Ln}(\mathrm{x})+108.64, \mathrm{R}^{2}=0.6307$.
In summary, spontaneous NK cell activity was not significantly different in AD subjects vs. normal control subjects at entry. The inhibitory effect of cortisol was significantly greater in patients with AD than in control subjects. NK activity was enhanced by IL-2 in all groups of subjects, albeit to a significantly lesser extent in patients with Fast Progression AD than in normal subjects, and compared to their cohorts with Slow Progression AD.

Taken together, our results suggest that patients with AD maintain NK activity and numbers within normal range. However, the cytotoxic activity of these cells is significantly more responsive to negative (cortisol) or positive physiologic modulators (IL-2) as the cognitive and behavioral symptoms associated with AD increase. These differences tend to accentuate with progression of the AD symptomatology.

Taken together, our data indicate that NK activity and modulation of NK activity by cortisol will become a simple and reliable tool to monitor progression of the disease. These assays will become important biomarkers of the disease since they can reveal, as we show, their use and accessibility for risk assessment, early diagnosis and follow-up of patients with $\mathrm{AD}$. Indeed, by performing the simple test of monitoring NK sensitivity to cortisol and to IL-2 modulation in vitro at entry, reliable predictions of the patients who would progress fast, vs. slowly to the more sever AD symptomatology is now possible. 


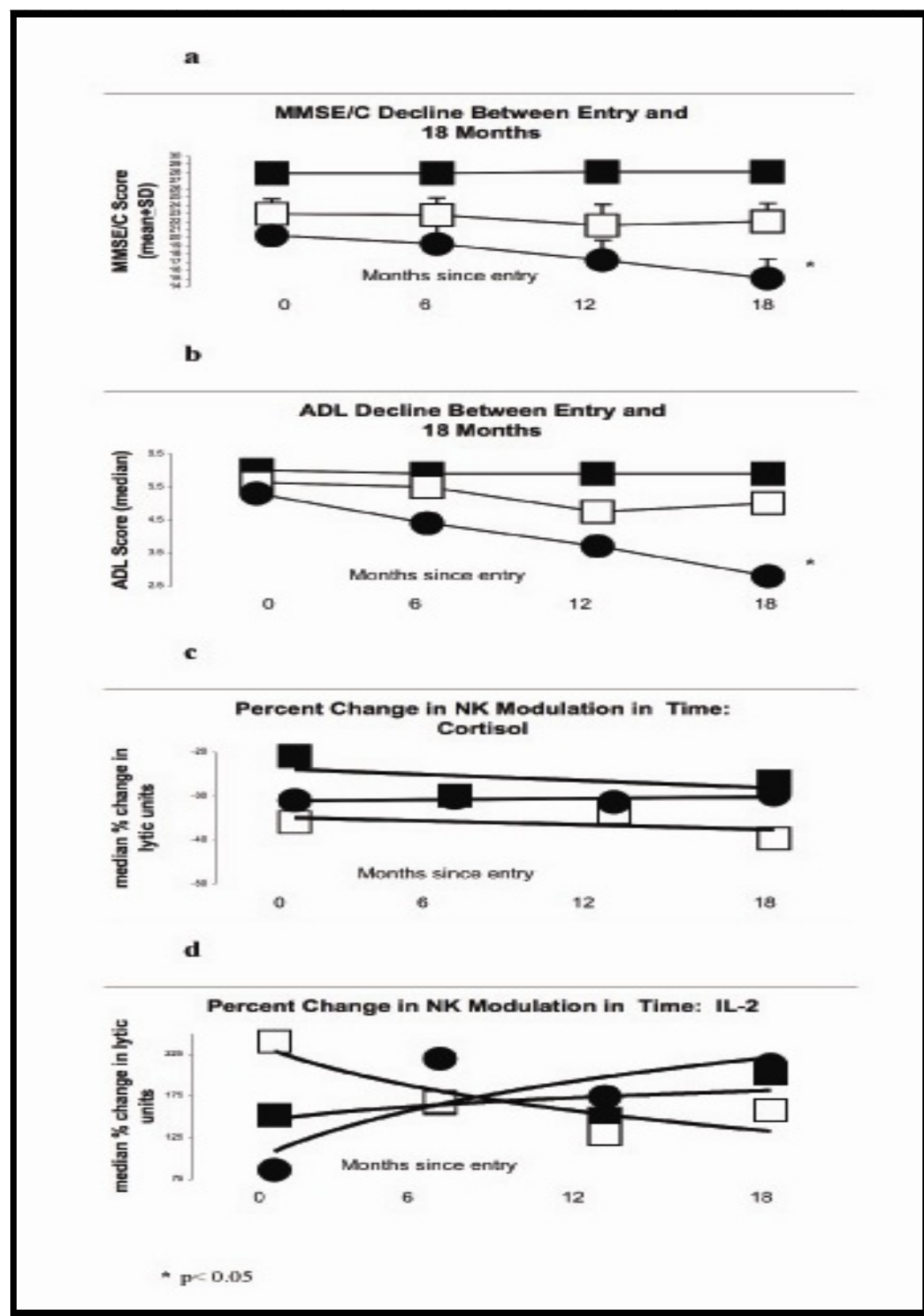

Figure 1: (a) Pattern of progression of AD symptoms in terms of the score for the Mini-Mental State Examination-corrected for educational level and ethnicity at 0 to 18 months. The data in the figure (mean + SD) show that the score on the MMSE-C dropped significantly faster in patients with Fast Progression AD ( $<<0.05)$, compared to their cohorts with Slow Progression AD. (closed squares: control subjects; open squares: patients with Slow Progression AD; closed circles: patients with Fast Progression AD); (b) Pattern of progression of $\mathrm{AD}$ symptoms in terms of the score for the Activities of Daily Living (ADL) (data presented as median values). (closed squares: control subjects; open squares: patients with Slow Progression AD; closed circles: patients with Fast Progression AD); (c) Pattern of change of the modulatory response of NK activity in time to cortisol among normal controls and patients with Slow Progression and Fast Progression AD at six month intervals between entry and 18 month; (d) Pattern of change in NK modulation by IL2 among normal controls and patients with Slow Progression and Fast Progression AD at six month intervals between entry and 18 month. (data are presented as median group percent change from baseline untreated cells to cells treated with the physiological modulator). (closed squares: control subjects; open squares: patients with Slow Progression AD; closed circles: patients with Fast Progression AD)

ISSN 0973-2063 


\section{Bioinformation}

\section{www.bioinformation.net}

\section{Current Trends}

\section{Acknowledgment:}

This work was supported in part by Fondazione Cassa di Risparmio di Cuneo; Fondazione Cassa di Risparmio di Saluzzo; Wilshire Rotary Foundation of Los Angeles; NIDDKD P50 DK 64539; and the Alzheimer's Association. The Authors want to thank Daniela Baraban of the Flowcytometry Laboratory at Ospedale S.Luigi, Orbassano for her valuable contribution to this study.

\section{References:}

[01] H. Fillit \& J. Cummings, J. Manag care Interface, 13:51 (2000) [PMID: 10747691]

[02] J. L. Cummings, Clin Cornerstone, 3:27 (2001) [PMID: 11432120]

[03] F. Chiappelli, et al., Evid Based Complement Alternat Med., 3:411 (2006) [PMID: 17173104]

[04] E. Esumi, et al., Acta Neurol Scand., 84:65 (1991) [PMID: 1927262]
[05] F. Chiappelli et al., Alzheimer's Disease: New Frontiers for the XXI Century. In Trends in Alzheimer's Disease Research, Nova Science Publisher, 233 (2006)

[06] M. Fiala, et al., Eur J Clin Invest., 32:360 (2002) [PMID: 12027877]

[07] M. Fiala, et al., J Alzheimer's Dis., 7:221 (2005) [PMID: 16006665]

[08] R. G. Masera, et al., Psychoneuroendocrinol., 27:447 (2002) [PMID: 11911998]

[09] A. Dovio, et al., Int J Obes Relat Metab Disord., 28:894 (2004) [PMID: 15208649]

[10] C. Korzeniewski \& D. M. Callewaert, J. Immun. Methods, 64:313(1983) [PMID: 6199426]

[11] H. F. Pross, et al., J. Clin. Immunol., 1:51(1981) [PMID: 7334070]

Edited by P.Kangueane Citation: Prolo et al., Bioinformation 1(9): 363-366 (2007) License statement: This is an open-access article, which permits unrestricted use, distribution, and reproduction in any medium, for non-commercial purposes, provided the original author and source are credited. 